\title{
Correlation between preoperatively predicted and postoperatively observed renal function using an imaging-based approach: A retrospective cohort study
}

\author{
JINGCHAO LIU, CHUANXIN TIAN, ZHAOCUN ZHANG, GUANWEN ZHOU, \\ BENKANG SHI, HAIFENG ZHAO and XIANZHOU JIANG \\ Department of Urology, Qilu Hospital of Shandong University, Jinan, Shandong 250012, P.R. China
}

Received December 18, 2019; Accepted January 22, 2020

DOI: 10.3892/ol.2020.11584

\begin{abstract}
The aim of the present study was to preoperatively predict renal function following partial nephrectomy (PN) using an imaging-based approach and to examine the correlation between preoperatively predicted and postoperatively observed renal function in the study cohort. A total of 128 consecutive patients who underwent PN between May 2015 and March 2018 and had available clinical data were included in this study. A hand-scripting method was used to estimate the defected volume (Vdef) from preoperative computerized tomography scans, whereas a cylindrical method was used to obtain preoperative renal volume (Vpre). The function index (FI) was proposed as a new term to estimate preserved parenchyma percentage following PN. The FI was defined as $\mathrm{f}=($ Vpre-Vdef $) /$ Vpre for the operated kidney and adjusted as FI=0.5 x $(f+1)$ for the bilateral kidneys. The estimated glomerular filtration rates (GFRs) before surgery, one day after surgery and $\sim 12$ months after surgery were calculated using the Modification of Diet in Renal Disease Study equation. The GFR rate after PN was predicted by multiplying the preoperative GFR by the FI. The predictive role of the FI was further tested using multiple linear regression and correlation analyses. The median FI in the present study was $94 \%$ for unilateral kidney surgery and adjusted to $97 \%$ for bilateral kidneys. Linear correlation analysis revealed that the predicted
\end{abstract}

Correspondence to: Dr Xianzhou Jiang or Dr Haifeng Zhao, Department of Urology, Qilu Hospital of Shandong University, 107 Wenhua Xi Road, Jinan, Shandong 250012, P.R. China

E-mail: jiangxianzhou123@126.com

E-mail: zhaohaifengzhuzhi@126.com

Abbreviations: PN, partial nephrectomy; WIT, warm ischemia time; CT, computerized tomography; FI, function index; GFR, glomerular filtration rate; MDRD, modification of diet in renal disease; PADUA, preoperative aspects and dimensions used for anatomy

Key words: GFR, PN, CT, warm ischemia
GFR significantly correlated with the observed immediate postoperative GFR $\left(\mathrm{R}^{2}, 0.594\right)$ and observed late postoperative GFR $\left(R^{2}, 0.828\right)$. In multivariate regression analysis, preoperative GFR $(\mathrm{P}<0.01)$ and warm ischemic time $(\mathrm{P}<0.01)$ were identified as independent determinants of the immediate postoperative renal function, whereas only $\mathrm{FI}(\mathrm{P}<0.01)$ and preoperative GFR $(\mathrm{P}<0.01)$ were identified as independent determinants of late renal function after PN. The preoperatively predicted renal function using an imaging-based approach had a significant positive correlation with the postoperatively observed renal function. The FI estimated from the preoperative diagnostic images in the present study was identified as an independent determinant of long-term renal function after PN.

\section{Introduction}

Partial nephrectomy (PN) is recommended as the best treatment for localized renal cell carcinoma due to improved renal function outcomes compared with radical nephrectomy $(1,2)$. Owing to intraoperative ischemic injury and excision of the renal parenchymal unit, renal function may decrease following PN. Recent mechanistic studies have revealed that acute kidney injury after PN may contribute to chronic kidney disease development and increase the risk of cardiovascular disease $(3,4)$. A number of studies into the predictors of renal function after PN have been conducted, and determinants associated with immediate or late renal function after PN have been well established (5-7). The quality (preoperative renal function) and quantity (quantity of preserved renal parenchyma during PN) of the kidney have been discovered as independent factors affecting renal function after PN (6-8). Warm ischemia time (WIT) has been identified to be an independent factor only for short-term renal function after $\mathrm{PN}$ and did not seem to influence long-term renal function $(6,8,9)$.

A number of studies have been conducted to predict renal function after PN on the basis of preoperative renal function, WIT and preserved parenchymal percentage (7,9-12). The preserved parenchyma percentage has become an important focus of research, and the corresponding measurement is mainly achieved by one of the following methods: i) Simultaneous analysis of preoperative and postoperative computerized tomography (CT) scans using advanced 
volumetric software $(7-9,11)$; ii) intraoperative estimation of preserved parenchyma percentage by surgeons $(10,12)$; and iii) assessment of the removed parenchyma volume by subtracting the preoperative tumor volume from the intraoperative specimen volume (13). These methods for quantitative measurement of the kidney after PN have been proven to be feasible and may be used to predict renal function after PN. However, the disadvantage of the previous studies was that the preserved parenchyma percentage was estimated or measured mainly from postoperative or intraoperative data, which were not available before PN during the treatment decision-making process. Thus, the purpose of the present study was to preoperatively predict renal function after PN with an imaging-based approach and to examine the correlation between preoperatively predicted and postoperatively observed renal function in the analyzed cohort. By using the function index (FI), the ability to estimate the preserved parenchyma percentage preoperatively, and its role in postoperative renal function were investigated.

\section{Materials and methods}

Patient population. After obtaining approval from the Qilu Hospital Review Board, the data of 128 patients who had undergone laparoscopic PN between May 2015 and March 2018 were retrospectively analyzed in the present study. All patients were followed-up by reviewing their electronic record or by telephone for at least 1 year, in order to obtain the following data: The serum creatinine levels measured $\sim 1$ week before PN, on the first day after PN, and $\sim 1$ year after PN. A total of $86(67.2 \%)$ patients were male and the median age (range) was 54.7 (24-79) years. All PNs were performed by two experienced surgeons (Professor Jiang and Professor Shi) using the standard technique, which are well described in previous studies $(14,15)$. A renal artery clamp was used to obtain a clear field during the operation. All patients were evaluated on the basis of the following inclusion criteria: i) Treatment for a single renal tumor with a pathological diagnosis of clear cell carcinoma; ii) bilateral symmetrical kidneys; iii) approach of laparoscopic PNs with the warm ischemic technique; and iv) available data on serum creatinine levels before PN, 1 day after PN, and $\sim 12$ months after PN. Creatinine levels were measured at the Qilu Hospital laboratory. Estimated glomerular filtration rate (GFR) was calculated using the Modification of Diet in Renal Disease (MDRD) equation (16). Various clinical data, including age, sex, body mass index, Preoperative Aspects and the Dimensions Used for Anatomy (PADUA) score (17), and WIT were also included in the present study.

Volumetric measurement. Standard contrast-enhanced CT scans with multi-slice CT system (Somatom Force; Siemens Healthineers) were routinely performed preoperatively. The slice thickness in the scans was $1.0 \mathrm{~mm}$. The artery phase was used for relevant identification and further measurement. Fig. 1 illustrates the measurement methodology for the volumetric analysis in the present study. The estimated defected volume consisted of two elements, the endophytic tumor volume and extra-damaged volume due to suturing or ischemia injury during PN. Preoperative kidney volume (Vpre) was measured from an analogical cylinder as presented in Fig. 1B and C, which was suggested by previous studies $(7,18)$. Preoperative renal volume was defined as $\operatorname{Vpre}=\pi \times(d / 2)^{2} \times h$, where $d$ is the renal diameter and $h$ is the renal height. The variable $h$ was calculated by multiplying the slice thickness $(1.0 \mathrm{~mm})$ by the number of cross sections, including the uppermost and lowermost borders of the kidney. Once the uppermost and lowermost cross sections were determined, the middle section of the kidney was also identified; on the middle section, one line perpendicular to the hilum and another line parallel to the hilum were drawn, passing through the center point, to calculate the value of $d$ as follows: $D=\left(d_{1}+d_{2}\right) / 2$ (Fig. 1C). Theoretically, the intraoperative defected volume included the endophytic tumor volume and extra-damaged parenchymal volume (Fig. 1A). The extra-damaged parenchymal volume consisted of the parenchyma removed to guarantee negative margins and the parenchyma that exhibited ischemic necrosis during the suturing procedure. Estimated defected volume (Vdef) was measured as presented in Fig. 1D and E. The defected volume was estimated during PN from the preoperative CT scans on the basis of the calculus theory; Vdef can be viewed as an irregular ellipsoid, which is made up of a number of small cylinders. The cylinder volume equals the product of $\mathrm{S}$ and $\mathrm{H}(\mathrm{V}=\mathrm{S} \times \mathrm{H})$, where $\mathrm{S}$ indicates basal area of the cylinder and $\mathrm{H}$ indicates the height of the cylinder. All the cylinder volumes were added to obtain the defected volume as follows: $\operatorname{Vdef}=\left(S_{1}+S_{2}+S_{3}+\ldots+S_{n}\right) \times h$, where h corresponds to the slice thickness $(1.0 \mathrm{~mm})$. The total defected areas on different cross sections were manually outlined by experienced surgeons. On each cross section, the estimated defected area was projected to be $5 \mathrm{~mm}$ larger compared with the normal tumor margins, depending on the surgeon's experience (Fig. 1E). The defected area could be easily calculated using a Syngo Studio simple CT viewer (Siemens Medical Solutions).

FI definition. FI was defined as the estimated preserved renal parenchyma percentage after PN. The estimated postoperative volume of the operated kidney was calculated by subtracting the defected volume from the preoperative kidney volume (Vpost=Vpre-Vdef). Thus, the preserved parenchyma percentage of the operated kidney was calculated as follows: $\mathrm{f}=($ Vpre-Vdef $) /$ Vpre. Vpre and Vdef were measured using the aforementioned method, and the whole procedure did not require any advanced volumetric software. In the cohort of the present study, all included patients had bilaterally symmetrical kidneys; thus, the preserved parenchyma percentage was adjusted as: $\mathrm{FI}=0.5 \mathrm{x}(1+\mathrm{f})$.

Renal function analysis. Preoperative GFR was calculated from the serum creatinine level measured $\sim 1$ week before PN. Immediate postoperative GFR was calculated from the creatinine level measured on the first day after PN. Late postoperative GFR was calculated from the creatinine level measured $\sim 1$ year after PN. All calculations were based on the MDRD equation. Predicted GFR was calculated by multiplying the preoperative GFR with the FI (predicted GFR=preoperative GFR x FI). Correlations between the predicted and immediate postoperative GFRs and that between the predicted and late GFRs were subsequently analyzed. 
A

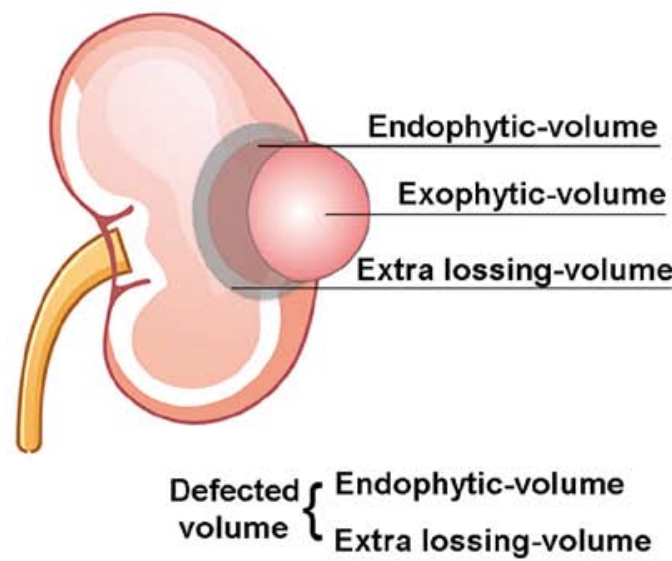

B

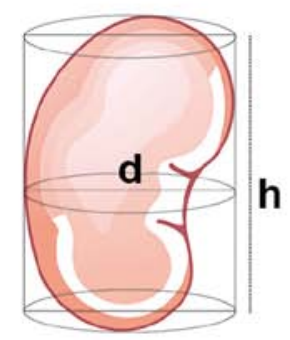

h

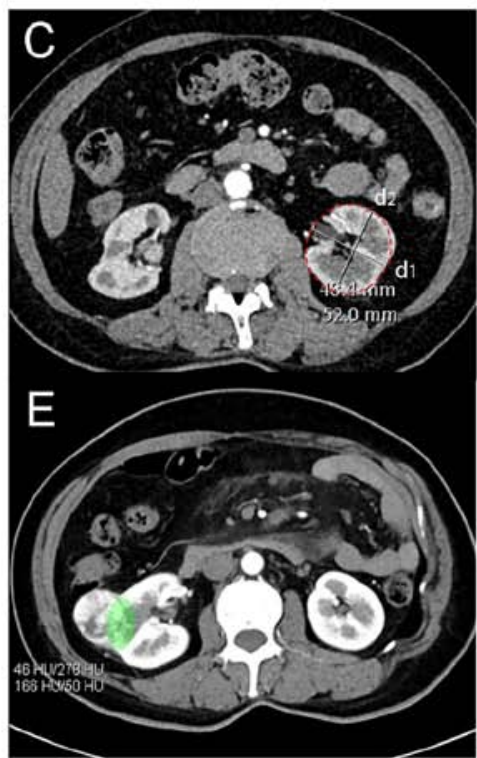

Figure 1. FI measurement methodology. (A) The estimated defected volume included the endophytic tumor and extra-damaged volumes. (B) The Vpre was measured using the cylinder method and was calculated as Vpre $=\pi \times(d / 2)^{2} \mathrm{x} h$. (C) On the middle section of the kidney, one line perpendicular to the hilum and another line parallel to the hilum were drawn, passing through the center point, to obtain the value of $d$, calculated as $d=\left(d_{1}+d_{2}\right) / 2$. $(D)$ The calculus theory was used in the measurement of defected volume, which can be viewed as an irregular ellipsoid that is made up of thousands of small cylinders. The cylinder volume is equal to the product of $\mathrm{S}$ and $\mathrm{h}(\mathrm{V}=\mathrm{S} \times \mathrm{h})$, and all the cylinder volumes were added to obtain the defected volume, calculated as $\mathrm{Vdef}=\left(\mathrm{S}_{1}+\mathrm{S}_{2}+\mathrm{S}_{3}\right.$ $\left.+\ldots+S_{n}\right) x h$. (E) On every cross section, the intraoperative defected area was projected at 5 mm larger compared with the normal tumor margins, depending on the surgeon's experience. Vpre, preoperative renal volume; $d$, renal diameter; h, corresponds to the slice thickness (1.0 mm); V, cylinder volume; $\mathrm{S}$, basal area of the cylinder; Vdef, defected volume.

Statistical analysis. Data are presented as means, with ranges or percentages as appropriate. The agreement between different measurers for defected volume was assessed using the Bland-Altman analysis. Multivariate linear regression analysis was performed to identify independent factors for FI, immediate postoperative GFR, and late GFR. Linear regression analysis was used to estimate the correlation between preoperatively predicted renal function and the postoperatively observed renal function. $\mathrm{P}<0.05$ was considered to indicate a statistically significant difference. Data were analyzed using SPSS 13.0 (SPSS, Inc.).

\section{Results}

Demographic, volumetric and functional data of the study cohort. Table I lists the basic demographic and clinical data of the study cohort. A total of 128 patients with available data were included in the present study, 86 (67.2\%) of whom were male. The median age was 55 years, and $60(46.9 \%)$ tumors were located in the left kidney. The median tumor diameter was 2.4 (range, $0.71-8.12$ ) $\mathrm{cm}$, and the median body mass index was 25.6 (range, 16.0-35.3) $\mathrm{kg} / \mathrm{m}^{2}$. The median PADUA score for renal tumors was 8.1 (range, 6-13), and the median WIT was 23.6 (range, 10-40) min. For estimating the renal function of the patients, the median serum creatinine level was 70.9 (range, 41-183) $\mu \mathrm{mol} / 1$ before surgery, 91.5 (44-290) $\mu \mathrm{mol} / 1$ on the first day after PN and 76.2 (42-190) $\mu \mathrm{mol} / 1 \sim 1$ year after PN. The median GFR based on the MDRD equation before surgery, on the first day after PN and $\sim 1$ year after PN were 97.6 (range, 31.7-162.7), 75.9 (range, 19.7-144.7) and 90.4 (range, 30.4-152.6) $\mathrm{ml} / \mathrm{min} / 1.73 \mathrm{~m}^{2}$, respectively. In addition, the median predicted GFR was 94.7 (range, 30.8-161.4) $\mathrm{ml} / \mathrm{min} / 1.73 \mathrm{~m}^{2}$.
In the volumetric analysis, the median kidney volume was 206 (range, 87.8-401.0) $\mathrm{ml}$, and the median estimated defected volume was 13.1 (range, 0.43-70.44) $\mathrm{ml}$. The median FI for an operated kidney was 0.94 (range, 0.67-1.00), and the median adjusted FI for the bilateral kidney was 0.97 (range, 0.83-1.00). The estimated defected volume was manually outlined by two experienced surgeons. The bias between two measurers was $-0.52 \mathrm{ml}$ (95\% CI, -1.73-.69), determined by the Bland-Altman analysis. The measured outcomes of the two measurers demonstrated a strong positive correlation $\left(\mathrm{R}^{2}, 0.984 ; \mathrm{P}<0.01\right)$; thus, there was a good agreement between the two measurers (Fig. 2). In addition, the postoperative pathological images demonstrated that the actual resected specimen boundary was consistent with the preoperative hand-scripted images, which both indicated a margin $\sim 5 \mathrm{~mm}$ larger compared with the normal tumor boundary (Fig. 3).

FI is an independent determinant of late GFR after PN. Multivariate linear regression analysis of the factors associated with FI revealed that only the operated kidney volume $(\mathrm{P}<0.01)$ and estimated defected volume $(\mathrm{P}<0.01)$ were significantly associated with FI (Table II). Correlation analysis demonstrated that predicted GFR correlated significantly with immediate postoperative GFR $\left(\mathrm{R}^{2}, 0.594\right.$; $\left.\mathrm{P}<0.01\right)$ and late GFR following PN ( $\mathrm{R}^{2}, 0.828$; $\left.\mathrm{P}<0.01\right)$ (Fig. 4).

In the multivariate regression analysis for identifying risk factors of renal function after $\mathrm{PN}$, preoperative GFR $(\mathrm{P}<0.01)$ and WIT $(\mathrm{P}<0.01)$ were independent determinants of immediate renal function following PN (Table III). FI did not serve a predictive role in determining immediate renal function after $\mathrm{PN}(\mathrm{P}=0.119)$. However, WIT did not exhibit a significant association with late renal function after $\mathrm{PN}(\mathrm{P}=0.287)$. FI $(\mathrm{P}<0.01)$ 
Table I. Patient demographic, operative and functional data.

\begin{tabular}{lc}
\hline Patient information & Overall \\
\hline No. of patients & 128 \\
No. of males (\%) & $86(67.2 \%)$ \\
Median age (range), years & $54.7(24-79)$ \\
No. of left kidney (\%) & $60(46.9 \%)$ \\
Median tumor largest diameter & $2.4(0.71-8.12)$ \\
(range), cm & \\
Median body mass index (range), kg/m ${ }^{2}$ & $25.6(16.0-35.3)$ \\
Median neutrophil-lymphocyte ratio & $2.59(0.07-42.14)$ \\
(range) & $8.1(6-13)$ \\
Median PADUA score (range) & $206.0(87.8-401.0)$ \\
Median kidney volume (range), ml & $13.1(0.43-70.44)$ \\
Median estimated defected-volume & \\
(range), ml & \\
FI & \\
Median operated kidney (range) & $0.94(0.67-1.00)$ \\
$\quad$ Median adjusted bilateral kidney (range) & $0.97(0.83-1.00)$ \\
Median ischemia (range), min & $23.6(10-40)$ \\
Median serum creatinine (range), $\mu \mathrm{mol} / \mathrm{l}$ & \\
Preoperative & $70.9(41-183)$ \\
1 day after surgery & $91.5(44-290)$ \\
Late & $76.2(42-190)$ \\
Median modification of diet in renal & \\
disease 2 GFR (range), ml/min/1.73 m² & \\
Preoperative & $97.6(31.7-162.7)$ \\
1 day after operation & $75.9(19.7-144.7)$ \\
$\quad$ Late & $90.4(30.4-152.6)$ \\
Median predicted GFR (range), & $94.7(30.8-161.4)$ \\
ml/min/1.73 m ${ }^{2}$ & \\
\hline & \\
\hline
\end{tabular}

FI, function index; GFR, glomerular filtration rate; PADUA, preoperative aspects and dimensions used for anatomy.

combined with preoperative GFR $(\mathrm{P}<0.01)$ were independent determinants of late GFR after PN in the multivariate linear regression analysis (Table III).

\section{Discussion}

The FI in the present study is a novel parameter developed from preoperative $\mathrm{CT}$ diagnostic images that indicates the estimated preserved renal parenchyma percentage after PN by experienced surgeons. The analysis in the present study shows that the GFR predicted preoperatively using the FI was positively correlated with the postoperatively observed GFR. A good interobserver agreement for FI was demonstrated, and FI was identified to be an independent risk factor of late GFR after PN. The ability of FI to preoperatively predict renal function after PN may greatly improve the clinical experience for both clinicians and patients.

Previous mechanistic research has demonstrated acute kidney injury and chronic kidney disease to be interconnected, both of which may contribute to cardiovascular disease (3).
A large number of studies investigating renal function after PN have been performed in recent years $(6,8,19-23)$. The risks of function reduction have been well established: The quality (preoperative renal function) and quantity (quantity of preserved renal parenchyma during $\mathrm{PN}$ ) of the kidney determine the late renal function after PN, and ischemia serves a secondary role in late functioning when the quantity of the kidney is included in the analysis $(6,18,21,24,25)$. The qualitative parameter of the kidney equates to preoperative GFR, and the quantitative parameter equates to the preserved renal parenchyma percentage.

The preserved renal parenchyma percentage has become a popular research topic in the past decade due to its significant influence on renal function after PN. Various volumetric methods of measurement of preserved parenchyma percentage have been developed to predict late renal function after PN $(7,9-12,18,26)$. In previous studies, three methods have been introduced to measure the preserved parenchyma percentage. The first method involves measurements on preoperative and postoperative $\mathrm{CT}$ scans to determine the preserved parenchyma percentage $(9,18)$. Simmons et al $(18)$ adopted the cylindrical volume methodology by analyzing both preoperative and postoperative CT images to measure preserved parenchyma percentage. Maria et al (9) used advanced volumetric software to analyze preoperative and postoperative CT images and found that preserved parenchyma percentage was highly correlated with kidney function. The second method involves measurements using intraoperative resected specimen and preoperative CT images $(13,26)$. Mitsui et al (26) recently introduced a volumetric method to predict postoperative renal function that did not depend on postoperative CT images. The third method involves intraoperative estimation of preserved parenchyma percentage by surgeons $(12,27)$. Previous research indicated that intraoperative estimation of preserved parenchyma by surgeons was as accurate as objective 3D measurements in predicting renal function (12). The aforementioned method helped urologists effectively predict renal function after PN and greatly improved the management in clinical settings with respect to renal function. However, the majority of previous studies relied on postoperative $\mathrm{CT}$ scans or intraoperative estimations to predict renal function, and these data are not available before PN. The present study aimed to estimate the preserved renal parenchyma percentage from preoperative CT images and identified FI as an effective predictor of postoperative renal function. FI, a preoperative parameter that reflects the estimated preserved parenchyma percentage, may further improve the clinical management and patient compliance. In addition, FI measurement relied on hand scripting using basic $\mathrm{CT}$ scan viewers, and advanced 3D volumetric software was not required. This measurement may be available in most primary institutions that cannot afford advanced 3D software.

Multivariate regression analysis revealed that tumor diameter was not significantly associated with FI in the present study. Only the operated kidney and estimated defected volumes were identified to be independent factors of FI. Previous studies have suggested that the relative volumetric measurement rather than the tumor diameter determined renal function outcomes following PN (28). Volumetric analysis was more accurate compared with tumor diameter 


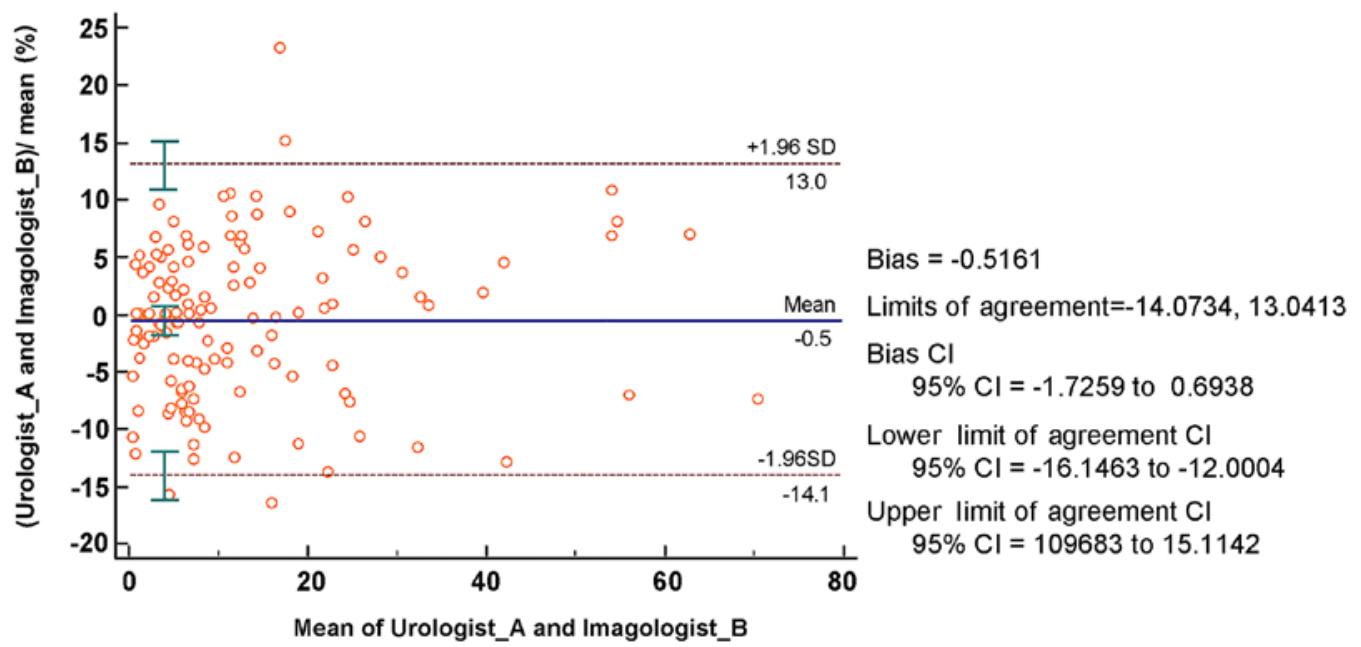

Figure 2. Bland-Altman analysis of the agreement between the two measurers. The mean outcome of the two measurers was plotted on the $x$-axis and the outcome of the difference/mean of the two measurers was plotted on the $y$-axis. The Bland-Altman plot revealed an arithmetic mean of- $0.51 \mathrm{ml}$ and a good agreement between the two measurers.
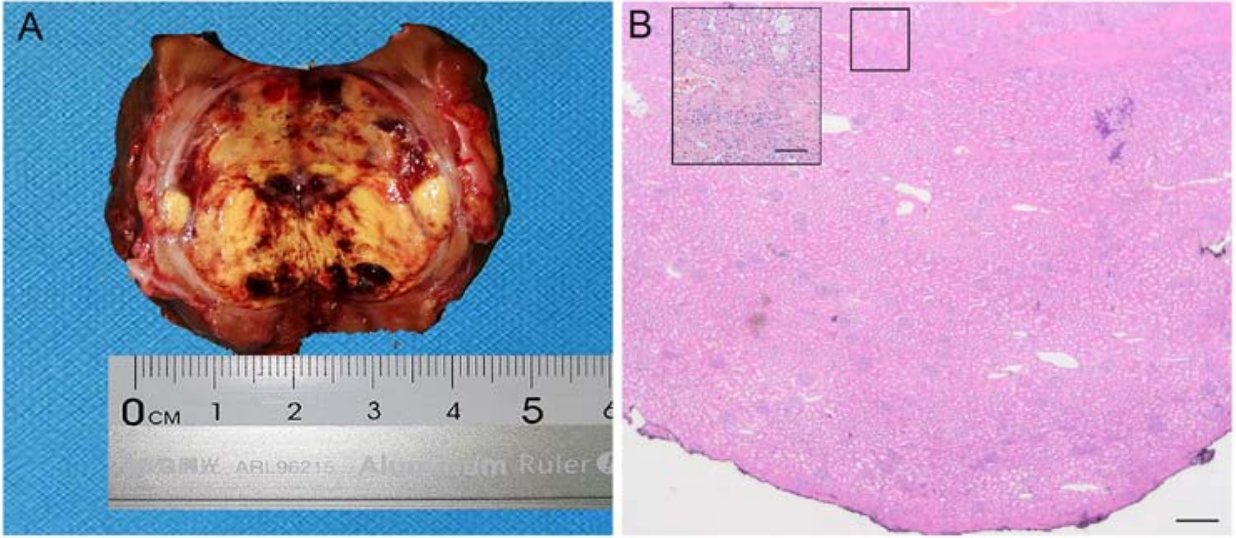

Figure 3. Pathological images demonstrating that the actual resected specimen boundary exhibited an $\sim 5 \mathrm{~mm}$ larger margin compared with normal tumor boundary. (A) Renal tumor including a margin $\sim 5 \mathrm{~mm}$ larger compared with the normal tumor boundary in the resected specimen. (B) Pathological images with hematoxylin-eosin staining with the peripheral parenchyma $\sim 5 \mathrm{~mm}$. Scale bars, $50 \mu \mathrm{m}$.
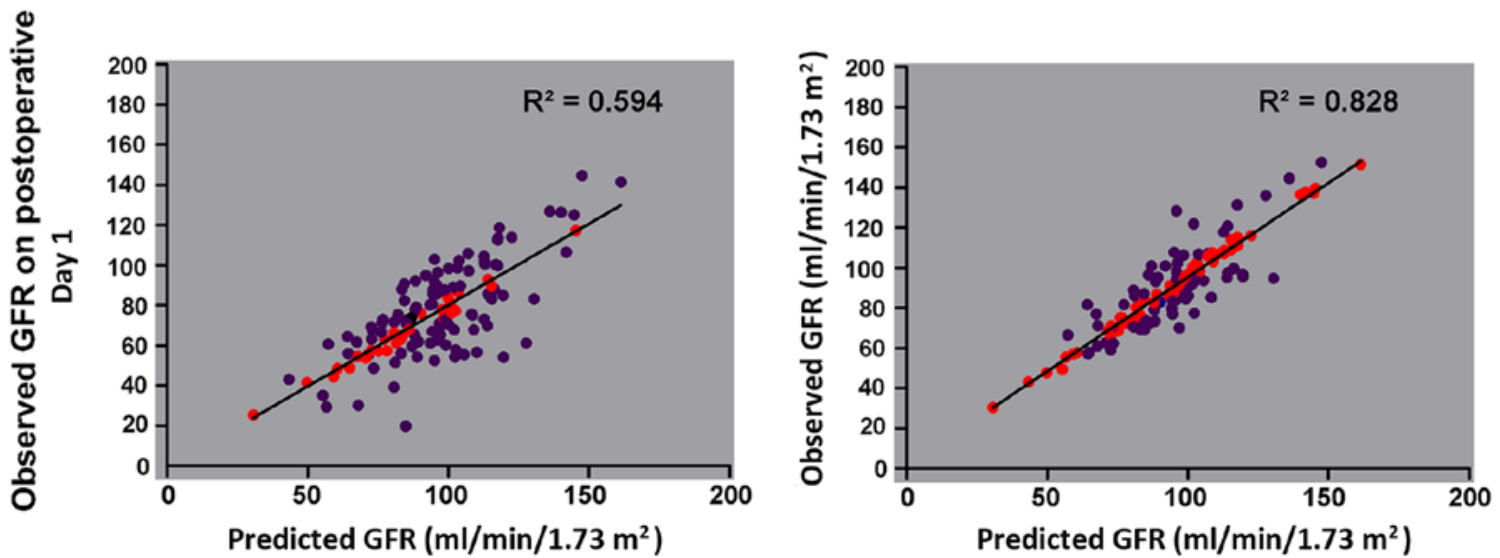

Figure 4. Predicted GFR significantly correlates with immediate postoperative GFR $\left(\mathrm{R}^{2}, 0.594 ; \mathrm{P}<0.01\right)$ and late GFR after partial nephrectomy $\left(\mathrm{R}^{2}, 0.828\right.$; $\mathrm{P}<0.01)$. GFR, glomerular filtration rate.

in predicting function outcomes in the present study. The operated kidney volume was measured using cylinder-based methodology (18), a procedure that can be easily performed using basic $\mathrm{CT}$ viewers. The estimated defected volume was calculated on the basis of infinitesimal calculus principles. Hand-scripting in the measurements may seem complex and 
Table II. Multivariate linear regression analysis of factors associated with FI.

\begin{tabular}{lrrr}
\hline Patient information & Coefficient \pm SE & T & P-value \\
\hline Age & $0.005 \pm 0.000$ & 0.155 & 0.877 \\
Sex & $-0.008 \pm 0.002$ & -0.291 & 0.771 \\
Left/right location & $-0.019 \pm 0.002$ & -0.689 & 0.492 \\
Body mass index & $0.021 \pm 0.000$ & 0.719 & 0.473 \\
Neutrophil-lymphocyte ratio & $-0.001 \pm 0.000$ & -0.023 & 0.982 \\
PADUA score & $-0.030 \pm 0.001$ & -0.943 & 0.348 \\
Warm ischemic time & $0.020 \pm 0.000$ & 0.679 & 0.499 \\
Preoperative GFR & $0.034 \pm 0.000$ & 1.021 & 0.310 \\
Tumor diameter & $-0.069 \pm 0.001$ & -1.743 & 0.084 \\
Operated kidney volume & $0.313 \pm 0.000$ & 9.489 & -23.951 \\
Estimated defected-volume & $-0.975 \pm 0.000$ & $<0.01^{\mathrm{a}}$ \\
\hline
\end{tabular}

${ }^{\mathrm{a}} \mathrm{P}<0.05$. FI, function index; GFR, glomerular filtration rate; PADUA, preoperative aspects and dimensions used for anatomy; T, Student's t-test.

Table III. Multivariate linear regression analysis of factors associated with eGFR after surgery.

A, GFR on postoperative day 1

\begin{tabular}{lrrr}
\hline Patient information & Coefficient \pm SE & T & P-value \\
\hline Age & $-0.098 \pm 0.097$ & -1.009 & 0.315 \\
Sex & $0.137 \pm 2.122$ & 0.065 & 0.948 \\
Left/right location & $-1.788 \pm 2.044$ & -0.875 & 0.384 \\
Body mass index & $-0.103 \pm 0.303$ & -0.341 & 0.733 \\
Neutrophil-lymphocyte ratio & $0.309 \pm 0.270$ & 1.145 & 0.254 \\
PADUA score & $0.679 \pm 0.752$ & 0.903 & 0.368 \\
Tumor diameter & $0.024 \pm 1.144$ & 0.021 & 0.984 \\
Preoperative GFR & $0.657 \pm 0.056$ & 11.639 & $<0.01^{\mathrm{a}}$ \\
Warm ischemic time & $-1.383 \pm 0.145$ & -9.554 & $<0.01^{\mathrm{a}}$ \\
\hline
\end{tabular}

B, Late GFR after PN

\begin{tabular}{lrrr}
\hline Patient information & Coefficient \pm SE & T & P-value \\
\hline Age & $-0.116 \pm 0.081$ & -1.439 & 0.153 \\
Sex & $3.103 \pm 1.770$ & 1.753 & 0.082 \\
Left/right location & $-1.652 \pm 1.705$ & -0.969 & 0.335 \\
Body mass index & $0.137 \pm 0.252$ & 0.542 & 0.589 \\
Neutrophil-lymphocyte ratio & $0.060 \pm 0.225$ & 0.266 & 0.791 \\
PADUA score & $-0.539 \pm 0.627$ & -0.946 & 0.346 \\
Tumor diameter & $0.531 \pm 0.954$ & 0.557 & 0.579 \\
Preoperative GFR & $0.851 \pm 0.047$ & 18.078 & -1.070 \\
Warm ischemic time & $-0.129 \pm 0.121$ & 3.776 & 0.287 \\
FI & $149.678 \pm 39.640$ & $<0.01^{\mathrm{a}}$ \\
\hline
\end{tabular}

${ }^{a} \mathrm{P}<0.05$. FI, function index; GFR, glomerular filtration rate; eGFR, estimated glomerular filtration rate; PADUA, preoperative aspects and dimensions used for anatomy; T, Student's t-test.

time-consuming; however, the majority of the renal tumors selected for PN were $<7 \mathrm{~cm}$ in diameter. The mean time of hand-scripting for tumors was $10 \mathrm{~min}$ in the present study.
Considering the predictive ability of FI for renal function following PN, hand-scripting may be an effective measurement modality. 
The serum creatinine level was routinely measured preoperatively and postoperatively as a follow-up parameter. However, serum creatinine level was not identified as an accurate indicator of renal function, as it may easily be influenced by muscle metabolism; instead, the estimated GFR was based on the MDRD equation. Although the estimated GFR was less accurate compared with a nuclide scan using ${ }^{125} \mathrm{I}$, the estimated GFR based on the MDRD equation has been proven to effectively reflect renal function (29). In addition, multiple nuclide scans using ${ }^{125} \mathrm{I}$ can be expensive and time-consuming for patients who have undergone PN.

In the present study, the preoperative GFR was multiplied by FI to obtain the predicted GFR. The linear correlation analysis identified that the predicted GFR had a strong linear correlation with the observed late GFR, whereas the linear correlation between the predicted GFR and immediate postoperative GFR was moderate. This result demonstrated that late renal function after PN depended on the qualitative (preoperative GFR) and quantitative measures (FI) of the kidney. By contrast, the immediate postoperative renal function may be influenced by other variables, including ischemic injury. The results of the multivariate analysis further confirmed that preoperative GFR and WIT were independent factors of immediate postoperative renal function, whereas FI and preoperative GFR were independent risk factors of late GFR after PN. During long-term follow-up, kidney nephrons recovered from ischemic injury and WIT lost its predictive role when FI was incorporated into the study. The results of the present study confirmed the conclusions of previous studies and demonstrated that the preserved renal parenchyma percentage may be estimated using the FI from preoperative CT images.

The mean FI for symmetrical bilateral kidneys was 0.97 in the present study. The estimated preserved renal parenchyma percentage in the present study was higher compared with that previously reported by Simmons et al as 93\% (18). The overestimation of the preserved parenchyma percentage may be due to several reasons. Firstly, FI was estimated from preoperative CT images, and this method did not take into account factors such as intraoperative ischemic injury, suturing necrosis and postoperative complications, which induced renal atrophy. The kidney could naturally atrophy over the follow-up period as a result of hypertension, diabetes and aging. Secondly, the utilization of preoperative imaging planning, intraoperative ultrasonography and experienced suturing techniques reduced the defected volume. In spite of the overestimated preserved parenchyma percentage, the significant predictive ability of FI revealed that it was a valuable predictive tool for clinical guidance.

The present study had various limitations, such as its retrospective nature, possible selection bias and limited samples selected from a single tertiary institution. In addition, all patients received the warm ischemia technique, as the hypothermic technique was rarely used at the institution where this study was performed. Additionally, the patients underwent laparoscopic PN for renal tumors, and further validation is required for open or robot-assisted PN. The FI in the present study relied on the subjective estimation of surgeons; however, it was indicated that the preoperatively predicted GFR using FI was highly correlated with postoperatively observed GFR in the study cohort. The renal function outcomes may be more informatively predicted if FI were to be routinely measured from preoperative diagnostic images in the future.

In conclusion, preoperatively predicted GFR using an imaging-based approach was positively correlated with postoperatively observed GFR. The FI estimated from preoperative diagnostic images served an independent role in predicting the long-term renal function following PN. Further attention should be paid to the preservation of functional parenchyma during PN surgeries in the future.

\section{Acknowledgements}

Not applicable.

\section{Funding}

This study was funded by the Key Research and Development Plan of Shandong Province (grant no. 2017GSF221005) and the Tai Shan Scholar Foundation (grant no. ts201511092).

\section{Availability of data and materials}

The datasets used and/or analyzed during the current study are available from the corresponding author on reasonable request.

\section{Authors' contributions}

JL was involved in study conception, and performed data collection and manuscript writing. CT performed the data analysis and data collection, and was involved in study conception. ZZ performed the data collection and analysis. $\mathrm{HZ}$ performed the data collection and was involved in study conception. GZ performed the data analysis, data collection and manuscript writing. BS performed the data analysis and was involved in study conception. XJ was involved in study conception, and performed data analysis and manuscript writing. All the authors read and approved the final manuscript.

\section{Ethics approval and consent to participate}

This study was approved by Qilu Hospital Review Board and was accordant with The Declaration of Helsinki. The patients provided written informed consent for participation.

\section{Patient consent for publication}

The patients provided written informed consent for publication.

\section{Competing interests}

The authors declare that they have no competing interests.

\section{References}

1. Ljungberg B, Albiges L, Abu-Ghanem Y, Bensalah K, Dabestani S, Fernández-Pello S, Giles RH, Hofmann F, Hora M, Kuczyk MA, et al: European association of urology guidelines on renal cell carcinoma: The 2019 update. Eur Urol 75: 799-810, 2019. 
2. Mir MC, Derweesh I, Porpiglia F, Zargar H, Mottrie A and Autorino R: Partial nephrectomy versus radical nephrectomy for clinical T1b and T2 renal tumors: A systematic review and meta-analysis of comparative studies. Eur Urol 71: 606-617, 2017

3. Chawla LS, Eggers PW, Star RA and Kimmel PL: Acute kidney injury and chronic kidney disease as interconnected syndromes. N Engl J Med 371: 58-66, 2014.

4. Bernstein A, Barry E, Fram EB, Sankin A, Kovac E and Stern JM: Does glomerular filtration rate at discharge after partial nephrectomy predict long-term glomerular filtration rate stability? J Endourol 33: 488-491, 2019.

5. Klatte T, Ficarra V, Gratzke C, Kaouk J, Kutikov A, Macchi V, Mottrie A, Porpiglia F, Porter J, Rogers CG, et al: A literature review of renal surgical anatomy and surgical strategies for partial nephrectomy. Eur Urol 68: 980-992, 2015.

6. Thompson RH, Lane BR, Lohse CM, Leibovich BC, Fergany A, Frank I, Gill IS, Blute ML and Campbell SC: Renal function after partial nephrectomy: Effect of warm ischemia relative to quantity and quality of preserved kidney. Urology 79: 356-360, 2012.

7. Simmons MN, Fergany AF and Campbell SC: Effect of parenchymal volume preservation on kidney function after partial nephrectomy. J Urol 186: 405-410, 2011.

8. Lane BR, Russo P, Uzzo RG, Hernandez AV, Boorjian SA, Thompson RH, Fergany AF, Love TE and Campbell SC: Comparison of cold and warm ischemia during partial nephrectomy in 660 solitary kidneys reveals predominant role of nonmodifiable factors in determining ultimate renal function. J Urol 185: 421-427, 2011.

9. Mir MC, Campbell RA, Sharma N, Remer EM, Simmons MN, Li J, Demirjian S, Kaouk J and Campbell SC: Parenchymal volume preservation and ischemia during partial nephrectomy: Functional and volumetric analysis. Urology 82: 263-268, 2013.

10. Chan AA, Wood CG, Caicedo J, Munsell MF and Matin SF: Predictors of unilateral renal function after open and laparoscopic partial nephrectomy. Urology 75: 295-302, 2010.

11. Song C, Bang JK, Park HK and Ahn H: Factors influencing renal function reduction after partial nephrectomy. J Urol 181: 48-54, 2009.

12. Tobert CM, Boelkins B, Culver S, Mammen L, Kahnoski RJ and Lane BR: Surgeon assessment of renal preservation with partial nephrectomy provides information comparable to measurement of volume preservation with 3 -dimensional image analysis J Urol 191: 1218-1224, 2014.

13. ZargarH, AkcaO, Autorino R,BrandaoLF,LaydnerH,Krishnan J, Samarasekera D, Stein RJ and Kaouk JH: Ipsilateral renal function preservation after robot-assisted partial nephrectomy (RAPN): An objective analysis using mercapto-acetyltriglycine (MAG3) renal scan data and volumetric assessment. BJU Int 115: 787-795, 2015.

14. Mottrie A, Borghesi M and Ficarra V: Is traditional laparoscopy the real competitor of robot-assisted partial nephrectomy? Eur Urol 62: 1034-1036; Discussion 1038-1039, 2012.

15. Porpiglia F, Volpe A, Billia M and Scarpa RM: Laparoscopic versus open partial nephrectomy: Analysis of the current literature. Eur Urol 53: 732-743, 2008.

16. Levey AS, Bosch JP, Lewis JB, Greene T, Rogers N and Roth D: A more accurate method to estimate glomerular filtration rate from serum creatinine: A new prediction equation. Modification of diet in renal disease study group. Ann Intern Med 130: 461-470, 1999

17. Ficarra V, Novara G, Secco S, Macchi V, Porzionato A, De Caro R and Artibani W: Preoperative aspects and dimensions used for an anatomical (PADUA) classification of renal tumours in patients who are candidates for nephron-sparing surgery. Eur Urol 56: 786-793, 2009
18. Simmons MN, Hillyer SP, Lee BH, Fergany AF, Kaouk J and Campbell SC: Functional recovery after partial nephrectomy: Effects of volume loss and ischemic injury. J Urol 187: 1667-1673, 2012.

19. Zhang Z, Zhao J, Velet L, Ercole CE, Remer EM, Mir CM, Li J, Takagi T, Demirjian S and Campbell SC: Functional recovery from extended warm ischemia associated with partial nephrectomy. Urology 87: 106-113, 2016.

20. Thompson RH, Frank I, Lohse CM, Saad IR, Fergany A, Zincke H, Leibovich BC, Blute ML and Novick AC: The impact of ischemia time during open nephron sparing surgery on solitary kidneys: A multi-institutional study. J Urol 177: 471-476, 2007.

21. Mir MC, Ercole C, Takagi T, Zhang Z, Velet L, Remer EM, Demirjian S and Campbell SC: Decline in renal function after partial nephrectomy: Etiology and prevention. J Urol 193: 1889-1898, 2015

22. Volpe A, Blute ML, Ficarra V, Gill IS, Kutikov A, Porpiglia F Rogers C, Touijer KA, Van Poppel H and Thompson RH: Renal ischemia and function after partial nephrectomy: A collaborative review of the literature. Eur Urol 68: 61-74, 2015.

23. Mir MC, Takagi T, Campbell RA, Sharma N, Remer EM, Li J, Demirjian S, Stein R, Kaouk J and Campbell SC: Poorly functioning kidneys recover from ischemia after partial nephrectomy as well as strongly functioning kidneys. J Urol 192: 665-670, 2014.

24. Kopp RP, Mehrazin R, Palazzi K, Bazzi WM, Patterson AL and Derweesh IH: Factors affecting renal function after open partial nephrectomy-a comparison of clampless and clamped warm ischemic technique. Urology 80: 865-870, 2012.

25. Ginzburg S, Uzzo R, Walton J, Miller C, Kurz D, Li T, Handorf E, Gor R, Corcoran A, Viterbo R, et al: Residual parenchymal volume, not warm ischemia time, predicts ultimate renal functional outcomes in patients undergoing partial nephrectomy. Urology 86: 300-305, 2015.

26. Mitsui Y, Sadahira T, Araki M, Maruyama Y, Nishimura S, Wada K, Kobayashi Y, Watanabe M, Watanabe T and Nasu Y: The 3-D volumetric measurement including resected specimen for predicting renal function after robot-assisted partial nephrectomy. Urology 125: 104-110, 2019.

27. Tobert CM, Takagi T, Liss MA, Lee H, Derweesh IH, Campbell SC and Lane B: Multicenter validation of surgeon assessment of renal preservation in comparison to measurement with 3D image analysis. Urology 86: 534-538, 2015.

28. Liss MA, DeConde R, Caovan D, Hofler J, Gabe M, Palazzi KL, Patel ND, Lee HJ, Ideker T, Van Poppel H, et al: Parenchymal volumetric assessment as a predictive tool to determine renal function benefit of nephron-sparing surgery compared with radical nephrectomy. J Endourol 30: 114-121, 2016.

29. Schwandt A, Denkinger M, Fasching P, Pfeifer M, Wagner C, Weiland J, Zeyfang A and Holl RW: Comparison of MDRD, CKD-EPI, and Cockcroft-Gault equation in relation to measured glomerular filtration rate among a large cohort with diabetes. J Diabetes Complications 31: 1376-1383, 2017.

(i) (-) This work is licensed under a Creative Commons Attribution-NonCommercial-NoDerivatives 4.0 International (CC BY-NC-ND 4.0) License. 